\title{
LCM LATTICES SUPPORTING PURE RESOLUTIONS
}

\author{
CHRISTOPHER A. FRANCISCO, JEFFREY MERMIN, AND JAY SCHWEIG
}

(Communicated by Irena Peeva)

\begin{abstract}
We characterize the $\mathrm{lcm}$ lattices that support a monomial ideal with a pure resolution. Given such a lattice, we provide a construction that yields a monomial ideal with that $\mathrm{lcm}$ lattice and whose minimal free resolution is pure.
\end{abstract}

\section{INTRODUCTION}

Understanding the minimal free resolutions of monomial ideals has been a deceptively difficult problem that has occupied researchers for over 50 years. In recent decades, combinatorial techniques have both helped to shed new light on methods for constructing minimal resolutions and illustrated some of the problems that arise. For example, while simplicial and cellular resolutions have proven useful in many situations, Velasco showed that there are monomial ideals whose minimal resolutions are not even supported on a CW-complex [V].

Nevertheless, combinatorial and topological methods still allow one to understand the minimal resolutions of monomial ideals at the cost of computing homologies. For example, formulas of Hochster [H] and Bayer, Charalambous, and Popescu $\mathrm{BCP}$ yield multigraded Betti numbers for any monomial ideal provided that one can compute the relevant homology groups for certain simplicial complexes. Gasharov, Peeva, and Welker introduce the lcm lattice in the context of resolutions in GPW. The combinatorial structure of the lcm lattice of an ideal determines its total Betti numbers and projective dimension. (See [Pe, Section 58] for background on lcm lattices.)

However, two ideals with isomorphic lcm lattices need not have the same graded Betti numbers. One particularly interesting type of free resolution is the class of pure resolutions, those for which at each step in the resolution, there is only a single shift; for background, see [Pe, Section 17]. Pure resolutions play a vital role in Boij-Söderberg theory, which decomposes free resolutions in an especially nice way. In this paper, we ask:

Question 1.1. When is a lattice the lcm lattice of a monomial ideal with a pure resolution?

We provide a complete answer to this question in Theorem 3.9, using a topological condition on the lattice that we call homologically monotonic (HM).

Theorem 1.2 (Theorem 3.9). Let $\Lambda$ be an atomic lattice. Then there exists an ideal $I$ with pure resolution such that $\operatorname{lcm}(I)=\Lambda$ if and only if $\Lambda$ is $H M$.

Received by the editors June 11, 2015 and, in revised form, July 1, 2015.

2010 Mathematics Subject Classification. Primary 06A07, 13D02. 
As we note in Remark 3.3, geometric and distributive (or, more generally, supersolvable) lattices are HM, and thus Theorem 3.9 encompasses a wide range of lattices. Using the proof of Theorem 3.9, given any lattice $\Lambda$ that is HM, we explain how to construct a monomial ideal with a pure resolution and lcm lattice $\Lambda$.

In Section 2, which follows the work of Phan [Ph] and Mapes [M], we review the necessary background on lcm lattices and describe the labeling techniques we will use in our proofs. The main results are in Section 3. We conclude in Section 4 with some discussion of possible future work.

\section{BACKGROUND AND NOTATION}

Throughout the paper, let $R=\mathbb{k}\left[x_{1}, x_{2}, \ldots, x_{n}\right]$ be the polynomial ring with sufficiently many variables over a field $\mathbb{k}$. Let $\Lambda$ denote a finite atomic lattice, defined below.

Definition 2.1. A finite poset $\Lambda$ is called a finite atomic lattice if it satisfies the following properties:

(1) Every subset $S \subseteq \Lambda$ has a unique least upper bound $\bigvee(S)$, called the join of $S$, and a unique greatest lower bound $\bigwedge(S)$, called the meet of $S$.

(2) Every element of $\Lambda$ is a join of atoms. (Recall that an atom is an element that covers the least element $\hat{0}$.)

Note that if $\Lambda$ is a finite atomic lattice, there are unique maximal and minimal elements $\hat{1}=\bigvee(\Lambda)$ and $\hat{0}=\bigwedge(\Lambda)$.

Finally, an element $v \in \Lambda \backslash\{\hat{1}\}$ is called meet-irreducible if it cannot be written as the meet of two larger elements.

Let $I$ be a monomial ideal with minimal generating set $G=\left(m_{1}, \ldots, m_{t}\right)$. Put $\Lambda=\{\mu: \mu=\operatorname{lcm}(S)$ for some $S \subseteq G\}$. Then $\Lambda$, ordered by divisibility, forms a finite atomic lattice. The generators of $I$ are the atoms, and $\hat{0}=1$. In this case, $\Lambda$ is called the lcm lattice of $I$, denoted $\operatorname{lcm}(I)=\Lambda$. See Figure 1 for an example.

Given a finite atomic lattice, there are two standard ways to associate monomials to some of its vertices (see $[\mathrm{M}]$ for details), and we are in the habit of referring to both associations as labelings. Unfortunately, the present paper will require these two labelings to interact with one another in complicated ways, so to avoid ambiguity we will avoid words like "labeling" in favor of "tags" for one association, and "multidegree" for the other.

Definition 2.2. A multigraded lattice is a pair $(\Lambda, \operatorname{mdeg})$, where $\Lambda$ is a finite atomic lattice and mdeg is a function from the vertices of $\Lambda$ to the monomials of $R$ which satisfies the properties below. (We refer to $\operatorname{mdeg}(v)$ as the multidegree of $v$.)

(1) Atoms of $\Lambda$ have incomparable multidegree.

(2) $\operatorname{mdeg}\left(w_{1} \vee w_{2}\right)=\operatorname{lcm}\left(\operatorname{mdeg}\left(w_{1}\right), \operatorname{mdeg}\left(w_{2}\right)\right)$.

(3) $\operatorname{mdeg}(\hat{0})=1$.

(4) For all $v, \operatorname{mdeg}(v)=\operatorname{lcm}(\operatorname{mdeg}(a): a \leq v$ and $a$ is an atom).

Observe that (4) is equivalent to (2) and (3).

Example 2.3. The lcm lattice of the ideal $I=\left(c^{2} d, a c d e, a^{2} b^{2}, a b c e\right)$ is shown in Figure 1. The multigrading function assigns each vertex its own multidegree; the lcm lattice together with this natural multigrading is a multigraded lattice. 


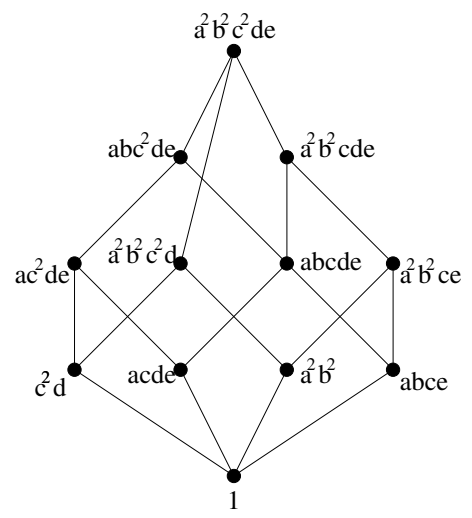

Figure 1. The (multigraded) lcm lattice of the ideal $I=\left(c^{2} d, a c d e, a^{2} b^{2}, a b c e\right)$.

Definition 2.4. A tagged lattice is a pair $(\Lambda, \tau)$, where $\Lambda$ is a finite atomic lattice and $\tau$ is a function from the vertices of $\Lambda$ to the monomials of $R$ which satisfies the properties below. (We refer to $\tau(v)$ as the tag of $v$.)

(1) If $v$ is meet-irreducible, then $\tau(v) \neq 1$.

(2) For every variable $x$, the set $\{v: x$ divides $\tau(v)\}$ forms a chain in $\Lambda$.

(3) $\tau(\hat{1})=1$.

Example 2.5. The lattice in Figure 2 is tagged. The underlying lattice $\Lambda$ is the same as in Figure 1. The tagging function $\tau$ assigns each unmarked vertex to the monomial 1 and each marked vertex to its marking. Observe that every meetirreducible vertex has a nontrivial tag, and that, while the variables $a, b$, and $c$ each appear in multiple tags, these tags always form a chain. We will see below that the tagged lattice of Figure 2 and the multigraded lattice of Figure 1 contain the same information.

There is a natural duality between tagged and multigraded lattices, given by the following constructions. (We use only Construction 2.6 in this paper but state Construction 2.7 for completeness.)

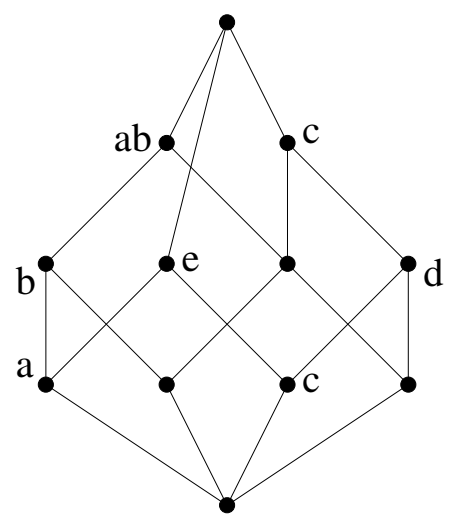

Figure 2. A tagged lattice. 
Construction 2.6 (Phan, Mapes). Suppose $(\Lambda, \tau)$ is a tagged lattice. For each vertex $v \in \Lambda$, set $\tau^{\mathrm{m}}(v)=\prod_{w \nsupseteq v} \tau(w)$. Then the pair $\left(\Lambda, \tau^{\mathrm{m}}\right)$ is a multigraded lattice.

Construction 2.7 (Mapes). Suppose $(\Lambda, \mathrm{mdeg})$ is a multigraded lattice. For each vertex $v \in \Lambda$ and each variable $x_{i}$, set $\ell_{i}(v)=\min _{w \not v}\left(\max \left(\ell: x_{i}^{\ell} \operatorname{divides} \operatorname{mdeg}(w)\right)\right)$ and $s_{i}(v)=\max \left(s: x_{i}^{s}\right.$ divides $\left.\operatorname{mdeg}(v)\right)$. Finally, set $\tau(v)=\prod x_{i}^{\ell_{i}(v)-s_{i}(v)}$. Then the pair $(\Lambda, \tau)$ is a tagged lattice.

Proposition 2.8 (Mapes). Constructions 2.6 and 2.7 are inverse bijections. In particular, there is a one-to-one correspondence between monomial ideals, multigraded lattices, and tagged lattices.

Proof. For the inverse bijection, see [M]. The correspondence to monomial ideals associates a monomial ideal to its (multigraded) lcm lattice and a multigraded lattice to the ideal generated by the multidegrees of its atoms.

The lattices of Examples 2.3 and 2.5 are associated to one another via Constructions 2.6 and 2.7 .

Example 2.9. Let $v \in \Lambda$ be as in Figure 3, where $\Lambda$ is the underlying lattice of our running examples. In Example 2.5, the vertices greater than or equal to $v$ have tags $b$ and $a b$, so the multidegree of $v$ is the product of the other tags, $\tau^{\mathrm{m}}(v)=(a)(c)(e)(d)(c)=a c^{2} d e$. In Example 2.3, the multidegree of $v$ is $a c^{2} d e$, which is not divisible by $b$. Since every vertex not less than or equal to $v$ has multidegree divisible by $b$, it follows that the tag of $v$ is divisible by $b$. Since there are incomparable elements with multidegrees divisible by the same power of every other variable, it follows that the tag of $v$ is in fact equal to $b$. Analyzing every other vertex in this manner, we see that the lattices of Examples 2.3 and 2.5 correspond under Constructions 2.6 and 2.7 .

Notation 2.10. Throughout the paper, we will study the (multigraded lattice and) monomial ideal coming from a tagged lattice via Construction 2.6. In order to avoid notational monstrosities like " $\operatorname{deg}(\operatorname{mdeg}(v))$ ", we refer to the multigrading as $\tau^{\mathrm{m}}$. Thus our multigraded lattices are $\left(\Lambda, \tau^{\mathrm{m}}\right)$, and in Example 2.9 we have $\tau^{\mathrm{m}}(v)=a c^{2} d e$ and $\operatorname{deg}\left(\tau^{\mathrm{m}}(v)\right)=5$.

The main algebraic motivation for studying lattices is that the Betti numbers of a monomial ideal are encoded in the poset structure of its lcm lattice.

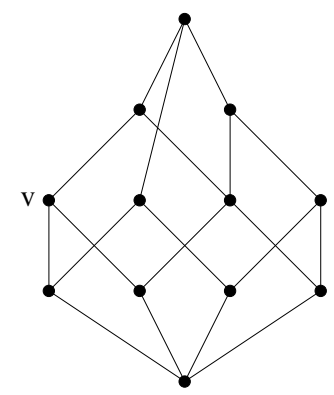

Figure 3. The lattice of the examples. 
For $x<y$ in a poset $P$, recall that the order complex of the open interval $(x, y)$ is the simplicial complex whose faces are chains in $P$ between $x$ and $y$. The following result is normally stated for the quotient $S / I$ rather than the ideal $I$; see, e.g., [Pe, Theorem 58.8].

Theorem 2.11 (Hochster's formula for lcm lattices). Let I be a monomial ideal, and let $\left(\Lambda, \tau^{m}\right)$ be its multigraded lcm lattice. Fix $v \neq \hat{0} \in \Lambda$, with $\tau^{m}(v)=\mu$. Then the multigraded Betti numbers of I with multidegree $\mu$ are given by the reduced homology of the order complex of the open interval $(\hat{0}, v)$ :

$$
b_{i, \mu}(I)=\tilde{H}_{i-1}(\hat{0}, v) .
$$

All other nontrivial multigraded Betti numbers are zero.

As the referee has pointed out, because we focus only on those multidegrees with nonzero Betti numbers, it is possible to rephrase all our major results in a slightly stronger form by replacing the lcm lattice with a coarser invariant called the Betti poset, which we introduce briefly below. We thank the referee for this insight.

Definition 2.12. Let $I$ be a monomial ideal. Then the Betti poset $B(I)$ is the set of shifts appearing in the minimal multigraded free resolution of $I$,

$$
B(I)=\left\{\mu: b_{j, \mu}(I) \neq 0 \text { for some } j\right\} .
$$

Alternatively, let $\Lambda$ be a finite atomic lattice. Then the Betti poset of $\Lambda$ is the subposet consisting of all elements supporting nontrivial interval homology,

$$
B(\Lambda)=\left\{v: \tilde{H}_{j}(\hat{0}, v) \neq 0 \text { for some } j\right\} .
$$

Clearly, ideals with isomorphic lcm lattices have isomorphic Betti posets. However, nonisomorphic lattices can have the same Betti poset. For example, TV, Example 5.4] shows that the edge ideals of a 4-cycle and a 4-path have combinatorially equivalent Betti posets, but distinct lcm lattices.

For more on the theory of Betti posets, see [CM2 and [TV]. For our purposes, the key result is that interval homology does not change when passing from the $\mathrm{lcm}$ lattice to the Betti poset. The following result is a consequence of CM2, Theorem 1.4] or [TV, Theorem 5.2].

Theorem 2.13 ([CM2, TV]). Let $\Lambda$ be a finite atomic lattice with Betti poset $B=B(\Lambda)$, and suppose $v$ is a vertex of $B$. Then for all $j$ we have an isomorphism $\tilde{H}_{j}^{B}(\hat{0}, v) \cong \tilde{H}_{j}^{\Lambda}(\hat{0}, v)$. That is, the interval homologies are the same whether we view the interval in $B$ or in $\Lambda$.

All the results in Section 3 that reference the Betti poset follow immediately from this theorem.

\section{LATTICES SUPPORTING A PURE RESOLUTION}

In this section, we prove our main theorem, characterizing the lattices supporting a pure resolution.

Definition 3.1. We say that $\Lambda$ is homologically monotonic or $H M$ if whenever $v<w \in \Lambda$ with $\tilde{H}_{i}(\hat{0}, v) \neq 0 \neq \tilde{H}_{j}(\hat{0}, w)$, then $i<j$.

For a nonnegative integer $k$, we say that $\Lambda$ is $k$-HM if the above condition holds whenever $i \geq k$. (So the notion of "HM" is the same as " 0 -HM".) 
Remark 3.2. We can expand the definition of $(k$-)HM to any poset with $\hat{0}$ by requiring the same condition on the open intervals. It follows from Theorem 2.13 that a lattice is HM if and only if its underlying Betti poset is, so in some sense this is properly viewed as a property of the Betti poset. We thank the referee for pointing out that a Betti poset is $\mathrm{HM}$ if and only if it is ranked by homological degree. There is no analogous statement for lattices.

Remark 3.3. If a lattice $\Lambda$ has a Cohen-Macaulay order complex, then it is HM: it follows from Reisner's criterion that for any $x$ in such a lattice, $\tilde{H}_{i}(\hat{0}, x)=0$ whenever $i$ is less than the dimension of the order complex of $(\hat{0}, x)$. But if $x, y \in \Lambda$ with $x<y$, the dimension of $(\hat{0}, x)$ is strictly less than the dimension of $(\hat{0}, y)$. Thus, if we have $\tilde{H}_{i}(\hat{0}, x) \neq 0 \neq \tilde{H}_{j}(\hat{0}, y)$, it follows that $i$ and $j$ are the dimensions of the order complexes of $(\hat{0}, x)$ and $(\hat{0}, y)$, respectively, so $i<j$.

As shellable lattices are Cohen-Macaulay, this observation shows that many classes of lattices are HM, such as supersolvable lattices (which include distributive lattices) and geometric lattices.

Lemma 3.4. Suppose that $\Lambda$ is $k-H M$, and let $v \in \Lambda$ with $\tilde{H}_{i}(\hat{0}, v) \neq 0$ for some $i \geq k$. Then, for all other $j \geq k$, we have $\tilde{H}_{j}(\hat{0}, v)=0$.

Proof. Assume without loss of generality that $i<j$. Now let $I$ be any ideal having lcm lattice $\Lambda$. (For example, $I$ could be the Phan ideal of $\Lambda$, obtained by tagging each meet-irreducible element with a distinct variable, tagging all other elements with 1, and then applying Construction 2.6.) Let

$$
\ldots \longrightarrow F_{j+1} \stackrel{\partial_{j+1}}{\longrightarrow} F_{j} \longrightarrow \ldots \longrightarrow F_{i} \longrightarrow \ldots \longrightarrow F_{0} \longrightarrow I \longrightarrow 0
$$

be a minimal free resolution of $I$. If $\tilde{H}_{j}(\hat{0}, v) \neq 0$, then by Hochster's formula, $F_{j+1}$ has a basis element $e$ with multidegree $\tau^{\mathrm{m}}(v)$. Then $\partial_{j+1}(e) \neq 0$, so we may write it as $\partial_{j+1}(e)=\sum m_{f} f$ for nonzero monomials $m_{f}$ and basis elements $f$ for $F_{j}$. Choose one such $f$; we have $\operatorname{mdeg}\left(m_{f} f\right)=\operatorname{mdeg}(e)=\tau^{\mathrm{m}}(v)$. It follows that mdeg $(f)$ divides $\tau^{\mathrm{m}}(v)$ and that there exists $w \in \Lambda$ with $\tau^{\mathrm{m}}(w)=\operatorname{mdeg}(f)$, so $w<v$. But now we have $\tilde{H}_{j-1}(\hat{0}, w) \neq 0$, and $j-1 \geq i$, contradicting the assumption that $\Lambda$ was $k$-HM.

Definition 3.5. Let $(\Lambda, \tau)$ be a tagged lattice. We say that $(\Lambda, \tau)$ is $k$-pure if whenever $\tilde{H}_{k}(\hat{0}, v) \neq 0 \neq \tilde{H}_{k}(\hat{0}, w)$ for two elements $v, w \in \Lambda$, then $\operatorname{deg}\left(\tau^{\mathrm{m}}(v)\right)=$ $\operatorname{deg}\left(\tau^{\mathrm{m}}(w)\right)$.

We also say that $\Lambda$ is $k$-pure if there exists a tagging $\tau$ so that $(\Lambda, \tau)$ is $k$-pure. Finally, we say that $(\Lambda, \tau)$ (resp. $\Lambda$ ) is pure if it is $k$-pure for all $k \geq 0$.

In order to quantify how far a given tagged lattice $(\Lambda, \tau)$ is from being $k$-pure, it helps to define the statistic $\delta_{k}$ as follows.

Definition 3.6. For a tagged lattice $(\Lambda, \tau)$, set

$$
\delta_{k}(\Lambda, \tau)=\max \left\{\operatorname{deg}\left(\tau^{\mathrm{m}}(v)\right): \tilde{H}_{k}(\hat{0}, v) \neq 0\right\}-\min \left\{\operatorname{deg}\left(\tau^{\mathrm{m}}(v)\right): \tilde{H}_{k}(\hat{0}, v) \neq 0\right\} .
$$

Note that $(\Lambda, \tau)$ is $k$-pure if and only if $\delta_{k}(\Lambda, \tau)=0$. 
Lemma 3.7. Let $(\Lambda, \tau)$ be a tagged lattice that is not $k$-pure, and suppose $\Lambda$ is $k$-HM. Then there exists a tagging $\tau_{1}$ with the following two properties:

(1) $\delta_{k}\left(\Lambda, \tau_{1}\right)<\delta_{k}(\Lambda, \tau)$.

(2) If $(\Lambda, \tau)$ is $\ell$-pure for some $\ell>k$, so is $\left(\Lambda, \tau_{1}\right)$.

Proof. Define two subsets $S, T \subseteq \Lambda$ by

$$
\begin{gathered}
S=\left\{v \in \Lambda: \tilde{H}_{k}(\hat{0}, v) \neq 0 \text { and } \operatorname{deg}\left(\tau^{\mathrm{m}}(v)\right) \text { is maximal }\right\}, \\
T=\left\{v \in \Lambda: \tilde{H}_{k}(\hat{0}, v) \neq 0 \text { and } \operatorname{deg}\left(\tau^{\mathrm{m}}(v)\right) \text { is not maximal }\right\} .
\end{gathered}
$$

Because $(\Lambda, \tau)$ is not $k$-pure, both $S$ and $T$ are nonempty. We claim that no two elements $v, w \in S \cup T$ can be comparable (i.e., $S \cup T$ is an antichain of $\Lambda$ ). Indeed, if $v, w \in S \cup T$ were comparable (suppose $v<w)$, we would have that both $\tilde{H}_{k}(\hat{0}, v)$ and $\tilde{H}_{k}(\hat{0}, w)$ are nonzero, but $k \nless k$, contradicting the assumption that $\Lambda$ is $k$-HM.

Now write $S=\left\{v_{1}, v_{2}, \ldots, v_{t}\right\}$, and let $x_{1}, x_{2}, \ldots, x_{t}$ be variables not appearing in the original tagging $\tau$. Define $\tau_{1}$ by $\tau_{1}(v)=\tau(v)$ if $v \notin S$ and $\tau_{1}\left(v_{i}\right)=\tau\left(v_{i}\right) x_{i}$ for $v_{i} \in S$. Now let $v \in S \cup T$. Since no two elements of $S \cup T$ are comparable, every element $w$ of $S \cup T$ satisfies $v \not \leq w$, except for $w=v$. It follows that for $v \in T$ we have

$$
\tau_{1}^{\mathrm{m}}(v)=\tau^{\mathrm{m}}(v) x_{1} x_{2} \cdots x_{t}
$$

whereas for $v_{i} \in S$ we get

$$
\tau_{1}^{\mathrm{m}}\left(v_{i}\right)=\tau^{\mathrm{m}}(v) x_{1} x_{2} \cdots \widehat{x_{i}} \cdots x_{t} .
$$

Thus, $\operatorname{deg}\left(\tau_{1}^{\mathrm{m}}(v)\right)=\operatorname{deg}\left(\tau^{\mathrm{m}}(v)\right)+t$ if $v \in T$, and $\operatorname{deg}\left(\tau_{1}^{\mathrm{m}}(v)\right)=\operatorname{deg}\left(\tau^{\mathrm{m}}(v)\right)+t-1$ if $v \in S$, meaning $\delta_{k}\left(\Lambda, \tau_{1}\right)=\delta_{k}(\Lambda, \tau)-1$. This proves $(1)$.

Now we show (2). Choose $v, w \in \Lambda$ with $\tilde{H}_{\ell}(\hat{0}, v) \neq 0 \neq \tilde{H}_{\ell}(\hat{0}, w)$. Since $(\Lambda, \tau)$ is $\ell$-pure, we have $\operatorname{deg}\left(\tau^{\mathrm{m}}(v)\right)=\operatorname{deg}\left(\tau^{\mathrm{m}}(w)\right)$.

We claim that no $u \in S$ can satisfy $v \leq u$. Since $\tilde{H}_{k}(\hat{0}, u) \neq 0 \neq \tilde{H}_{\ell}(\hat{0}, v)$, we could not have $v<u$ since $k>\ell$ would contradict our assumption that $\Lambda$ is $k$-HM. Similarly, by Lemma 3.4 we could not have $v=u$. Thus,

$$
\operatorname{deg}\left(\tau_{1}^{\mathrm{m}}(v)\right)=\operatorname{deg}\left(\tau^{\mathrm{m}}(v)\right)+|\{u \in S: v \not \leq u\}|=\operatorname{deg}\left(\tau^{\mathrm{m}}(v)\right)+|S|,
$$

and the same holds for $w: \operatorname{deg}\left(\tau_{1}^{\mathrm{m}}(w)\right)=\operatorname{deg}\left(\tau^{\mathrm{m}}(w)\right)+|S|$. So we have $\operatorname{deg}\left(\tau_{1}^{\mathrm{m}}(v)\right)=$ $\operatorname{deg}\left(\tau_{1}^{\mathrm{m}}(w)\right)$, meaning $\left(\Lambda, \tau_{1}\right)$ remains $\ell$-pure.

Lemma 3.8. Let $(\Lambda, \tau)$ be a tagged lattice that is $\ell$-pure, and suppose $\Lambda$ is $k$-HM for some $k<\ell$. Then there is a tagging of $\Lambda$ so that the associated tagged lattice is both $k$-pure and $\ell$-pure.

Proof. If $(\Lambda, \tau)$ is $k$-pure, we're done. Otherwise, we have $\delta_{k}(\Lambda, \tau)>0$. We apply Lemma 3.7 obtaining a tagging $\tau_{1}$ so that $\left(\Lambda, \tau_{1}\right)$ is $\ell$-pure and $\delta_{k}\left(\Lambda, \tau_{1}\right)=\delta_{k}(\Lambda, \tau)-$ 1. If $\delta_{k}\left(\Lambda, \tau_{1}\right)>0$, we repeat the algorithm from Lemma 3.7 again, obtaining a tagging $\tau_{2}$ so that $\left(\Lambda, \tau_{2}\right)$ is $\ell$-pure and $\delta_{k}\left(\Lambda, \tau_{2}\right)=\delta_{k}\left(\Lambda, \tau_{1}\right)-1$. Continuing, we eventually obtain a tagging $\sigma=\tau_{j}$ (actually, $j=\delta_{k}(\Lambda, \tau)$ ) so that $\left(\Lambda, \tau_{j}\right)$ is $\ell$-pure and $\delta_{k}\left(\Lambda, \tau_{j}\right)=0$, meaning $\left(\Lambda, \tau_{j}\right)$ is also $k$-pure.

Theorem 3.9. Let $\Lambda$ be an atomic lattice. Then there exists an ideal I with pure resolution such that $\operatorname{lcm}(I)=\Lambda$ if and only if $\Lambda$ is $H M$. 
Proof. First, suppose that $\Lambda$ is not HM. Then there must be some $v, w \in \Lambda$ with $v<w$ and $\tilde{H}_{i}(\hat{0}, v) \neq 0 \neq \tilde{H}_{j}(\hat{0}, w)$ but $i \geq j$. If $I$ is any ideal with $\operatorname{lcm}(I)=\Lambda$, then $v$ and $w$ must correspond to monomials $m_{v}$ and $m_{w}$ with $\operatorname{deg}\left(m_{v}\right)<\operatorname{deg}\left(m_{w}\right)$. If $i=j$, then $I$ does not have a pure resolution. If $i>j$, then $b_{i+1}\left(m_{w}\right) \neq 0$. It follows that $b_{j+1}(\mu) \neq 0$ for some $\mu$ dividing $m_{w}$. Since $\mu$ divides $m_{v}$, it follows that $I$ does not have pure resolution.

Now suppose $\Lambda$ is HM, and fix a tagging $\tau$. Let $i$ be the greatest integer for which $(\Lambda, \tau)$ is not $i$-pure. By Lemma 3.8, we can construct a tagging $\tau_{1}$ so that $\left(\Lambda, \tau_{1}\right)$ is $k$-pure for all $k \geq i$. Now let $j$ be the greatest integer for which $\left(\Lambda, \tau_{1}\right)$ is not pure (so that $j<i$ ). Again by Lemma 3.8, we can construct a tagging $\tau_{2}$ such that $\left(\Lambda, \tau_{2}\right)$ is $k$-pure for all $k \geq j$. Continuing in this way, we eventually obtain a tagging $\tau_{r}$ so that $\left(\Lambda, \tau_{r}\right)$ is pure. Thus $I=I\left(\Lambda, \tau_{r}\right)$ is an ideal with pure resolution satisfying $\operatorname{lcm}(I)=\Lambda$.

A slightly stronger version of Theorem 3.9 replaces the lattice with its Betti poset.

Corollary 3.10. Let $B$ be a poset that occurs as the Betti poset of a monomial ideal (or, equivalently, of a lattice). Then there exists an ideal with pure resolution and Betti poset $B$ if and only if $B$ is $H M$.

Example 3.11. If we apply the algorithm implicit in the proof of Theorem 3.9 to the running example of Section 2, the tagged lattice of Figure 2 becomes the new tagged lattice shown in Figure 4. The new variables in the tags are shown with capital letters.

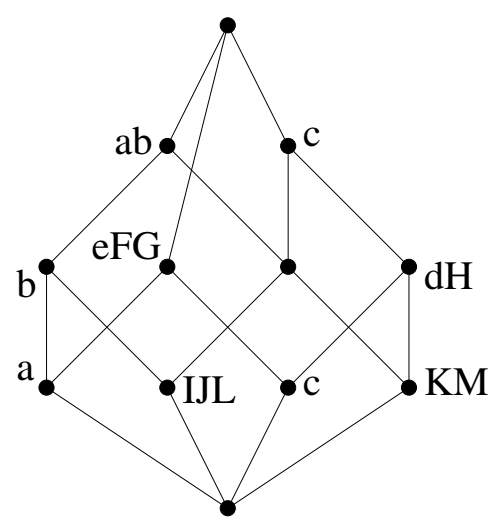

Figure 4. The purification of the tagged lattice in Figure 2.

The corresponding monomial ideal is

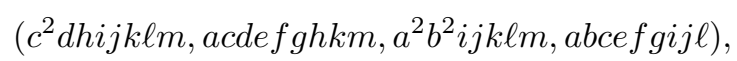

which has a pure resolution in degrees 9, 13, and 16 .

Observe that we could have done this with fewer variables by using pure powers for our new tags. Then the resulting monomial ideal is $\left(c^{2} d g h^{3} i^{2}, a c d e f^{2} g i^{2}\right.$, $a^{2} b^{2} h^{3} i^{2}$, abce $\left.f^{2} h^{3}\right)$. We could have used fewer variables still, if we were willing to reuse pre-existing variables for our new tags, but the result is highly nonunique. 


\section{Remarks And Questions}

Say that a monomial ideal is purifiable if there exists another ideal with pure resolution and the same lcm lattice. Then $I$ is purifiable if and only if its lcm lattice is HM, and many important classes of monomial ideals are purifiable. It is natural from an algebraic perspective to seek out classes of ideals which are (or are not) purifiable.

Example 4.1. Recall that a monomial ideal is called Scarf if it is resolved by its Scarf complex; see [BPS] for the construction. Then, following the same reasoning as in Remark 3.3. Scarf ideals are purifiable: an element of the lcm lattice supports homology only if it appears in the supporting complex, and then only in the dimension of the corresponding cell. Since the "generic monomial ideals" defined in BPS] are all Scarf, it follows that purifiability is very common.

A larger, if less well-studied, class of purifiable ideals is the rigid monomial ideals introduced by Clark and Mapes [CM]. These are the ideals whose resolutions have only scalar automorphisms; in particular, Scarf ideals are rigid. Clark and Mapes show that rigidity is equivalent to a stronger condition on the multigraded Betti numbers than being HM.

Definition $4.2(\underline{\mathrm{CM}})$ ). A monomial ideal $I$ is rigid if it satisfies the following conditions:

(R1) Every multigraded Betti number $b_{i, m}(I)$ is equal to either zero or one.

(R2) Multidegrees with the same nonzero Betti number are incomparable: If $m$ divides $m^{\prime}$, then for all $i$ at least one of $b_{i, m}(I)$ and $b_{i, m^{\prime}}(i)$ is equal to zero.

Applying Hochster's formula Theorem 2.11] it is clear that rigid ideals have HM $\mathrm{lcm}$ lattices and thus are purifiable. In fact, purifiability is equivalent to condition (R2):

Proposition 4.3. A finite atomic lattice $\Lambda$ is $H M$ if and only if it satisfies the following condition: Suppose $v<w \in \Lambda$. Then for all $i$, at least one of $\tilde{H}_{i}(\hat{0}, v)$ and $\tilde{H}_{i}(\hat{0}, w)$ is zero.

Proof. If $\Lambda$ is HM, the given condition is immediate.

For the converse, the idea is the same as the proof of Lemma 3.4, Let $I$ be any ideal with lcm lattice $\Lambda$, and let $m_{v}$ and $m_{w}$ be the corresponding multidegrees. Consider a minimal free resolution of $I$. The $i^{\text {th }}$ free module has a generator in multidegree $m_{v}$ if and only if $b_{i, m_{v}}(I) \neq 0$, if and only if $\tilde{H}_{i-1}\left(\hat{0}, m_{v}\right) \neq 0$. Now if $\Lambda$ is not HM, there exist numbers $i \geq j$ and vertices $v<w \in \Lambda$ with $b_{i, m_{v}}(I)$ and $b_{j, m_{w}}(I)$ both nonzero. Choose a generator for the $i^{\text {th }}$ free module with multidegree $m_{v}$, and apply the differential map. The result is an $R$-linear combination of generators for the $(i-1)^{\text {th }}$ free module, each with multidegree dividing $m_{v}$. Pick one such generator and repeat; after $(i-j)$ steps we will have found a generator for the $j^{\text {th }}$ free module with multidegree strictly dividing $m_{w}$, violating the given condition.

In another direction, we can attempt to generalize the Scarf condition to a larger class of supporting complexes. 
Question 4.4. Suppose that a minimal resolution of $I$ is supported by a topological object $X$ with nice structure. Are there conditions on $X$ (e.g., being a regular cell complex) which allow us to conclude that $I$ is purifiable?

Our intuition here is along the lines of Remark 3.3 and Example 4.1 each cell should correspond to a vertex in the lcm lattice, supporting homology only in its top dimension. The problem is that the face poset of $X$ is not just a subset of the lcm lattice of $I$ : faces can share multidegree, and incomparable faces can have comparable multidegrees.

We note that both purifiability of an ideal and homological monotonicity of a lattice are characteristic-dependent phenomena.

Example 4.5. Let $I=(a b c, a b f, a c e, a d e, a d f, b c d, b d e, b e f, c d f, c e f)$ be the StanleyReisner ideal of the minimal triangulation of the real projective plane, and let $\Lambda$ be the $\mathrm{lcm}$ lattice of $I$. In characteristic other than two, $I$ has a pure resolution, so $\Lambda$ is HM. However, in characteristic two, $I$ has multiple Betti numbers in multidegree $a b c d e f$. This corresponds to the open interval $(\hat{0}, \hat{1})$ having nonzero first and second homology in $\Lambda$, which means that $\Lambda$ is not HM.

Remark 4.6. If $\Lambda$ is any lattice with characteristic-dependent homology, it cannot be HM in the special characteristics: looking at the resolution of any corresponding ideal, the consecutive cancellations that occur when passing from the special characteristic to characteristic zero correspond to a trivial complex in some (fixed) multidegree. The vertex of $\Lambda$ with that multidegree consequently has two nonzero homologies in the special characteristic.

We conclude with an intriguing question suggested by the referee.

Question 4.7. How can we identify pure ideals that are as small as possible? In particular, is there some nice class of lcm lattices for which the Phan ideal is already pure and the algorithm in the proof of Theorem 3.9 is unnecessary?

\section{ACKNOWLEDGMENTS}

This work was partially supported by grants from the Simons Foundation (\#199124 to Christopher Francisco and \#202115 to Jeffrey Mermin).

\section{REFERENCES}

[BCP] Dave Bayer, Hara Charalambous, and Sorin Popescu, Extremal Betti numbers and applications to monomial ideals, J. Algebra 221 (1999), no. 2, 497-512, DOI 10.1006/jabr.1999.7970. MR1726711 (2001a:13020)

[BPS] Dave Bayer, Irena Peeva, and Bernd Sturmfels, Monomial resolutions, Math. Res. Lett. 5 (1998), no. 1-2, 31-46, DOI 10.4310/MRL.1998.v5.n1.a3. MR1618363 (99c:13029)

[CM] Timothy B. P. Clark and Sonja Mapes, Rigid monomial ideals, J. Commut. Algebra 6 (2014), no. 1, 33-52, DOI 10.1216/JCA-2014-6-1-33. MR3215560

[CM2] Timothy B. P. Clark and Sonja Mapes, The Betti poset in monomial resolutions. Preprint. arXiv:1407.5702.

[GPW] Vesselin Gasharov, Irena Peeva, and Volkmar Welker, The lcm-lattice in monomial resolutions, Math. Res. Lett. 6 (1999), no. 5-6, 521-532, DOI 10.4310/MRL.1999.v6.n5.a5. MR.1739211 (2001e:13018)

[H] Melvin Hochster, Cohen-Macaulay rings, combinatorics, and simplicial complexes, Ring theory, II (Proc. Second Conf., Univ. Oklahoma, Norman, Okla., 1975), Dekker, New York, 1977, pp. 171-223. Lecture Notes in Pure and Appl. Math., Vol. 26. MR0441987 (56 \#376) 
[M] Sonja Mapes, Finite atomic lattices and resolutions of monomial ideals, J. Algebra 379 (2013), 259-276, DOI 10.1016/j.jalgebra.2013.01.005. MR.3019256

[Pe] Irena Peeva, Graded syzygies, Algebra and Applications, vol. 14, Springer-Verlag London, Ltd., London, 2011. MR2560561 (2011j:13015)

$[\mathrm{Ph}]$ Jeffry Phuquoc Phan, Order properties of monomial ideals and their free resolutions, ProQuest LLC, Ann Arbor, MI, 2006. Thesis (Ph.D.)-Columbia University. MR.2708612

[TV] Alexandre Tchernev and Marco Varisco, Modules over categories and Betti posets of monomial ideals, Proc. Amer. Math. Soc. 143 (2015), no. 12, 5113-5128.

[V] Mauricio Velasco, Minimal free resolutions that are not supported by a CW-complex, J. Algebra 319 (2008), no. 1, 102-114, DOI 10.1016/j.jalgebra.2007.10.011. MR2378063 (2008j:13028)

Department of Mathematics, 401 Mathematical Sciences Building, Oklahoma State University, Stillwater, OKLAhoma 74078

E-mail address: chris.francisco@okstate.edu

URL: http://math.okstate.edu/people/chris/

Department of Mathematics, 401 Mathematical Sciences Building, Oklahoma State University, Stillwater, OKLAhoma 74078

E-mail address: mermin@math.okstate.edu

$U R L:$ http://math.okstate.edu/people/mermin/

Department of Mathematics, 401 Mathematical Sciences Building, Oklahoma State University, Stillwater, OKLAhoma 74078

E-mail address: jay.schweig@okstate.edu

URL: http://math.okstate.edu/people/jayjs/ 